\title{
A comprehensive review of current treatments and therapies for idiopathic pulmonary arterial hypertension
}

Danielle Lancaster ${ }^{1}$, Fuad Zeid, MD ${ }^{1}$

\section{Author Affiliations:}

1. Marshall University Joan C. Edwards School of Medicine, Huntington, West Virginia

The authors have no conflicts of interest to disclose.

\section{Corresponding Author:}

Danielle Lancaster

Marshall University Joan C. Edwards School of Medicine

Huntington, West Virginia

Email: lancaster19@live.marshall.edu 


\section{Abstract}

There are several classifications of Pulmonary Hypertension, one of them being Pulmonary Arterial Hypertension (PAH). In cases in which the cause of PAH is unknown, the disease is referred to as Idiopathic Pulmonary Arterial Hypertension (IPAH). IPAH is an extremely rare disease, making it difficult to treat. However, over the past few decades, treatments have expanded and improved. Treatments commonly used in clinical practice include one or more of the following: calcium channel blockers, prostacyclin derivatives, endothelin receptor antagonists, phosphodiesterase 5 inhibitors, guanylate cyclase stimulators, and in extreme cases lung transplants (single or double) or heart and lung transplants. The aim of this paper is to review the treatments and therapies available for IPAH because new information has been added after FDA approval of new medications. This article also raises ideas of possible future clinical trials to look at how these medications would work in a combination therapy.

\section{Keywords}

Idiopathic Pulmonary Arterial Hypertension, IPAH, primary pulmonary hypertension, PPH

\section{Introduction}

Blood is carried from the heart to the lungs via the pulmonary arteries. Pulmonary hypertension (PH) occurs when the blood pressure in these arteries increases. PH can be classified into five groups, according to the World Health Organization Table 1. ${ }^{1}$ One of the groups is pulmonary arterial hypertension. PAH is divided into subgroups based on etiology and associated treatment options. The one of interest in this paper is idiopathic pulmonary arterial hypertension (IPAH), which is the diagnosis when the cause cannot be determined. ${ }^{2}$ Accompanying the increased blood pressure in the arteries, PAH is also classified by the vascular remodeling of the small pulmonary arteries. ${ }^{3}$ Because this remodeling causes damage, gradual resistance in the vasculature occurs often leads to right heart failure. This condition is often fatal because of its rapid progression. 
Table 1: Pulmonary Hypertension Classifications. [Based off Baliga R.S. et al. 2011, Figure 1.]

\begin{tabular}{|c|c|c|c|c|}
\hline $\begin{array}{l}\text { 1. Pulmonary Arterial } \\
\text { Hypertension (PAH) }\end{array}$ & $\begin{array}{l}\text { 2. Pulmonary } \\
\text { Hypertension } \\
\text { with Left Heart } \\
\text { Disease }\end{array}$ & $\begin{array}{l}\text { 3. Pulmonary } \\
\text { Hypertension Due to } \\
\text { Lung Diseases and/or } \\
\text { Hypoxia }\end{array}$ & $\begin{array}{l}\text { 4. Chronic } \\
\text { Thromboembolic } \\
\text { Pulmonary } \\
\text { Hypertension }\end{array}$ & $\begin{array}{l}\text { 5. Pulmonary } \\
\text { Hypertension } \\
\text { with Indistinct, } \\
\text { Multi Factorial } \\
\text { Mechanisms }\end{array}$ \\
\hline $\begin{array}{l}1.1 \text { Idiopathic (IPAH) } \\
\text { 1.2 Heritable/Familial (FPAH) } \\
\text { 1.2.1 BMPR2 } \\
\text { 1.2.2 ALK1, Endoglin } \\
\text { 1.2.3 Unknown } \\
\text { 1.3 Drug and Toxin-Induced } \\
\text { 1.4 Associated with (APAH) } \\
\text { 1.4.1. Connective Tissue } \\
\text { Disorders } \\
\text { 1.4.2. HIV infection } \\
\text { 1.4.3. Portal Hypertension } \\
\text { 1.4.4. Congenital Heart } \\
\text { disease } \\
\text { 1.4.5 Schistasamiasis } \\
\text { 1.4.6 Chronic Hemolytic } \\
\text { Anemia } \\
\text { 1.5 Persistent Pulmonary } \\
\text { Hypertension of the } \\
\text { Newborn }\{\text { PPHN }\} \\
\text { 1' Pulmonary Vena-occlusive } \\
\text { Disease }\{\text { PVOD }\} \text { and Pulmonary } \\
\text { Capillary Hemangiomatous }\{\text { PCH }\}\end{array}$ & $\begin{array}{l}2.1 \text { Systolic } \\
\text { Dysfunction } \\
\text { 2.2 Diastolic } \\
\text { Dysfunction } \\
2.3 \text { Valvular } \\
\text { Disease }\end{array}$ & $\begin{array}{l}\text { 3.1 Chronic Obstructive } \\
\text { Pulmonary Disease } \\
\text { (COPD) } \\
\text { 3.2 Interstitial Lung } \\
\text { Disease } \\
\text { 3.3 Other Pulmonary } \\
\text { Diseases with Mixed } \\
\text { Restrictive and } \\
\text { Obstructive Patten } \\
\text { 3.4 Sleep Disordered } \\
\text { Breathing } \\
\text { 3.5 Alveolar } \\
\text { Hyperventilation } \\
\text { Disorders } \\
\text { 3.6 Chronic Exposure of } \\
\text { High Altitude } \\
\text { 3.7 Developmental } \\
\text { Abnormalities }\end{array}$ & & $\begin{array}{l}5.1 \text { Hematological } \\
\text { Disorders } \\
5.2 \text { Systemic } \\
\text { Disorders } \\
\text { 5.3 Metabolic } \\
\text { Disorders } \\
\text { 5.4 Others }\end{array}$ \\
\hline
\end{tabular}

IPAH is uncommon with an incidence of occurrence in one in every one million patients. Clinical research opportunities are limited due to the rarity of this disease. ${ }^{2}$ This review article is aimed to describe the available treatment options of IPAH. Current medications prescribed to these patients include calcium channel blockers, endothelin receptor antagonists, vasodilators, etc. The fatal outcome of IPAH can be slowed but not stopped.

\section{Background}

\section{Diagnostic Process}

The first indications of PH include fatigue, dizziness, lethargy, and dyspnea. If a patient presents with these symptoms along with a chest pressure, chronic cough, shortness of breath, swollen legs, the process to confirm the diagnosis and causation begins. ${ }^{4}$ The diagnostic approach is similar within the five groups of PH. As with most diagnoses, the approach starts with a physical exam and patient history. Further screening is performed by use of an echocardiogram, which permits a noninvasive diagnostic method. To confirm the diagnosis the gold standard is performance of a right heart catheterization. Both the echocardiogram and right heart catheterization are used to measure pulmonary artery systolic pressure, but often agreement 
between the two is limited. If mean right heart catheterization pressure exceeds $25 \mathrm{mmHg}$, a diagnosis of PAH is suspected. ${ }^{4}$ Even if the pressure is determined to be low, it is not sufficient to exclude PAH as a possible diagnosis. Other indications to consider are right arterial or ventricular enlargement, systolic dysfunction, hypertrophy, etc. ${ }^{5}$

Other tests to confirm the diagnosis, potential causes, and determination of medication for PAH include blood tests, blood vessel test, chest x-ray, CT scan, MRI, pulmonary function tests, and ventilation/perfusion scan. The most common test used to evaluate the prognosis of a patient is the 6-minute walk test, which is also often the test used in determining the efficacy, during clinical trials, of treatments for diseases such as PAH. IPAH is a diagnosis of exclusion; therefore if the cause cannot be determined using a variety of tests, a diagnosis of IPAH is made. ${ }^{6}$ A simplified version of the diagnostic process, created using information from the Cleveland Clinic, can be seen in Figure 1. ${ }^{7}$

Figure 1. Outlines stages of the diagnostic process recommended by the Cleveland clinic. ${ }^{7} \mathrm{CT}$, computed tomography; ECG, electrocardiogram; HIV, human immunodeficiency virus; $\mathrm{PH}, \mathrm{PAH}$, pulmonary arterial hypertension; $\mathrm{TT}$, transthoracic; $\mathrm{VO}_{2}$, oxygen consumption.

Stage 1

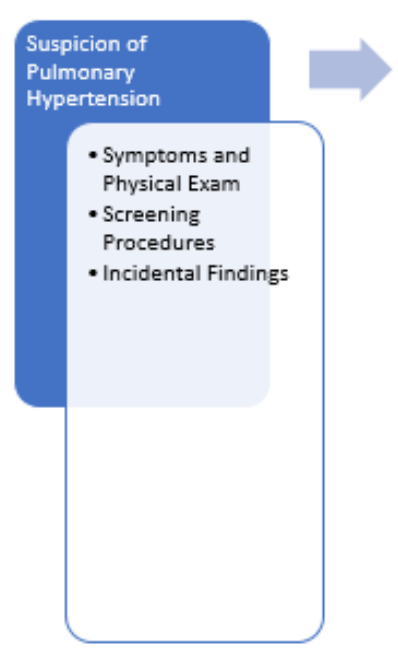

Stage 2

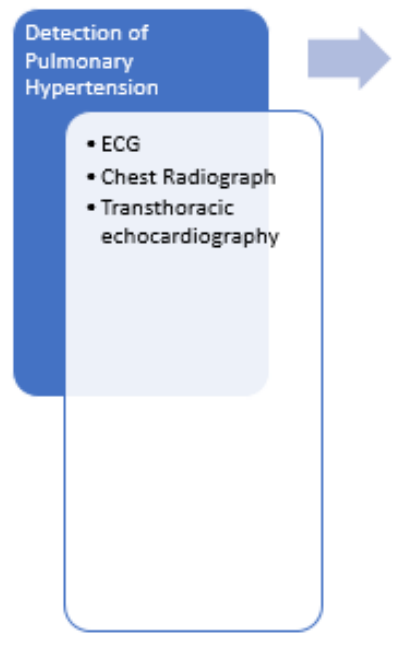

Stage 3

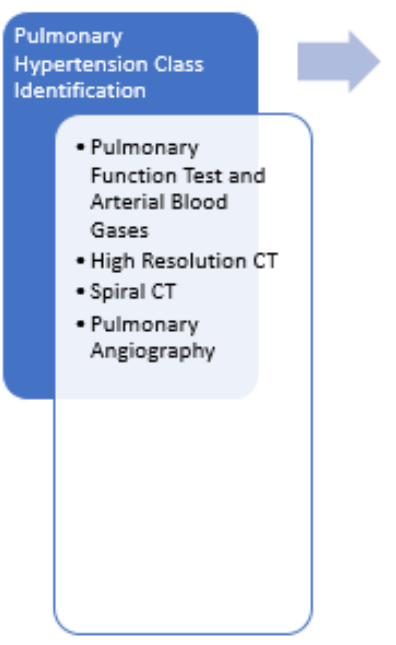

Stage 4

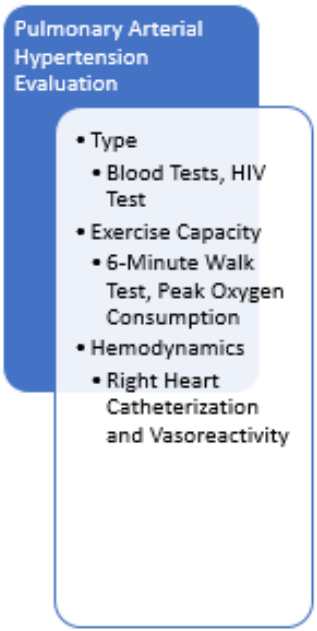

\section{Molecular Pathways Involved}

Currently, three pathways are implicated (Figure 2); these pathways include the endothelin (ET), prostacyclin, and NO pathways and are targets for the current drug therapies. Figure 2 was created using information from several others. ${ }^{8-11}$ Approved PAH therapies include ET receptor antagonists (ERAs), prostacyclin derivatives, phosphodiesterase-5 (PDE5) inhibitors, and a soluble guanylate cyclase (sGC) stimulator. 
Figure 2: The known pathways in PAH Targeted pathways and the therapies that affect them. Green arrows denote an increase in the intracellular concentration, red arrows indicate blockage of a receptor, inhibition of an enzyme or a decrease in the intracellular concentration. $\mathrm{ET}=$ endothelin; PDE-5 = phosphodiesterase type 5; sGC= soluble guanylate cyclase. [Figure created using combined information from Burger, C.D et al. 2016, Zhang, Y. et al. 2012 and Humber, M. 2004]

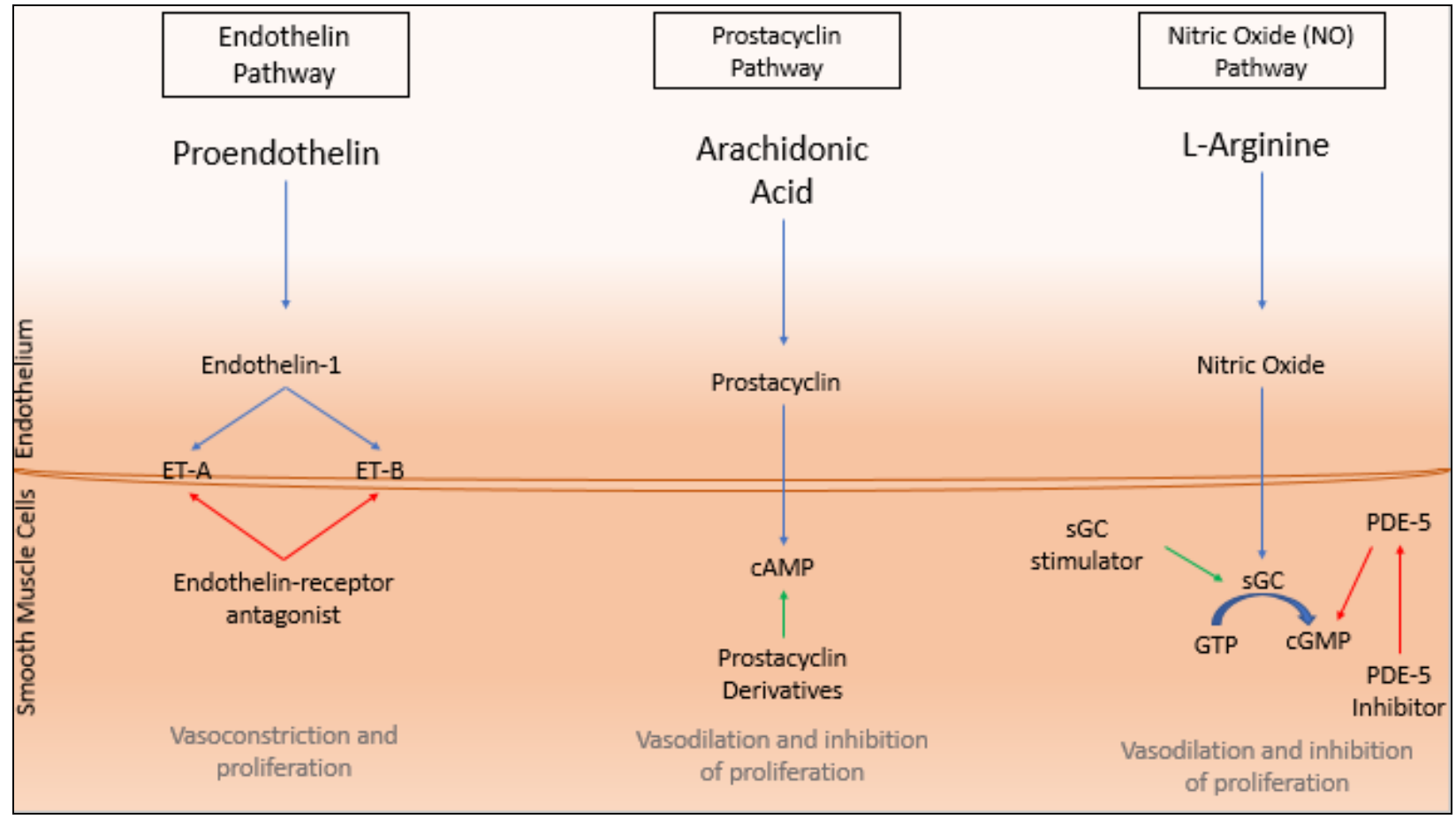

\section{Methods}

The articles were obtained using PubMed, Medline, google, and google scholar. Clinical trials were only included in this review article if the medication of interest was approved by the FDA for the use of IPAH. Within the previously mentioned databases multiple phrases were searched including: "idiopathic pulmonary arterial hypertension", "pulmonary arterial hypertension", "clinical trial AND IPAH", "treatments for idiopathic pulmonary arterial hypertension", etc. After finding treatment options for IPAH a google search was conducted to find FDA approved medications; once found, PubMed, Medline, and google scholar were used to find the clinical trials involved in these FDA approvals. Because IPAH is a rare disease, there was no restriction applied to the date of publication.

\section{Treatments and Therapies}

Up until 1996 the gold standard of treatment for IPAH, then called primary pulmonary hypertension, included the use of anticoagulants, calcium channel blockers (CCB), and/or the use of oxygen therapy. ${ }^{12}$ As of 2013, all other FDA approved medications fell into three categories: prostacyclin derivatives, endothelin receptor antagonists (ERA), phosphodiesterase 5 inhibitors (PDE-5). ${ }^{3}$ More recently, a new drug class, guanylate cyclase stimulators, was added to 
suggested treatments of IPAH. In severe cases in which medications are no longer efficacious, transplantations of lung(s) or heart and lungs are considered.

\section{Prostacyclin Derivatives}

Prostacyclins became of interest when the medical field discovered that levels were low in PAH patients. The discovery was made by Moncada, S. et. al. in $1976 .{ }^{13}$ Prostacyclins are vasodilators that inhibit platelet accumulation and have anti-proliferative properties. The three major prostacyclin derivatives used are epoprostenol, treprostinil, and iloprost. The recently approved medication Selexipag is included in this group as well.

Epoprostenol was first approved by the FDA in 1995. It is continuously administered intravenously using an infusion pump, due to its short half-life of only six minutes. Because of this, infusion interruption is a concern. ${ }^{5}$ Reservoir cartridges must be changed every eight hours (without the use of ice packs) because of the instability of the medication at room temperature. Even with the use of ice packs it must be changed every twenty-four hours. With the short half life and needing to change the reservoir cartridge, patients are under time constraints to get the infusion pump up and running again. Another concern is that since a catheter is required for continuous infusion for an extended length of time, complications such as infection of the line and thrombosis arise. The major side effects of epoprostenol include but are not limited to: headache, hypotension, anxiety, chest pain, nausea, and vomiting. ${ }^{12}$ Overall, epoprostenol has been shown to have survival benefits, as well as to improve IPAH symptoms and exercise capacity.

Almost a decade after the approval of epoprostenol, the FDA in 2002 approved treprostinil; it has side effects similar to that of epoprostenol with the addition of dizziness and jaw pain. Treprostinil has an extended half-life of about 4.5 hours, is chemically stable at room temperature, and has a $\mathrm{pH}$ that is neutral. ${ }^{12}$ It can be administered via infusion (intravenously or subcutaneously) or by inhaled formulations. Infusion site pain is a pragmatic association. Oral forms of treprostinil are currently under FDA review. But, missing oral doses could be potentially fatal and cause an abrupt return of symptoms. ${ }^{5,14}$

Three years after the approval of treprostinil, iloprost was approved by the FDA. Iloprost is an inhaled prostacyclin derivative, but inconveniently requires 6-9 times of dosing per day. Because of the side effects that occur with infusion therapies, inhaled versions were developed to help alleviate those side effects. Although inhaled versions are a safer alternative, the number of doses per day required is a challenge for patient compliance. In addition to the side effects experienced with epoprostenol and treprostinil, iloprost side effects include but are not limited to muscle cramps, back pain, insomnia, and some altered tastes. ${ }^{12}$

Ten years after iloprost hit the market selexipag was given the stamp of approval by the FDA in 2015. Selexipag is an oral medication that works by relaxing the muscles found in the blood vessel walls, which causes the blood vessels to dilate, allowing for the pressure to decrease. During the clinical trial stage it was shown to reduce hospitalization and slow progression of the disease. ${ }^{15}$ Side effects of selexipag are similar to the above mentioned: headache, muscle pain, diarrhea, jaw pain, nausea, vomiting, pain in extremities, (anemia), decreased appetite, and/or rash. 
These prostacyclin derivatives all have risks and benefits but the choice of mechanism of administration varies per case.

\section{Endothelin Receptor Antagonists (ERA)}

Endothelin receptor antagonists (ERA) inhibit endothelin-1. In all forms of PAH, endothein-1 is typically overexpressed. ET-1 is one of the most potent vasoconstrictors and induces smooth muscle cell proliferation. ET-1 acts through receptors ET-A and ET-B. In patients with PAH, elevated levels of ET-1 are commonly seen in the circulation and in the lungs. ${ }^{16}$

Three of the approved ERAs are bosentan, ambrisentan, and macitentan, which are all oral therapies. They have been shown to increase exercise tolerance and avoid clinical worsening. Because they are highly teratogenic, all ERA medications require monthly pregnancy tests in women of child bearing potential. Potential side effects for ERAs, in addition to the side effects mentioned for the procyclin derivatives, include: cold and flu like symptoms, urinary tract infection, and bronchitis. ${ }^{5}$

Liver functioning tests are mandatory monthly when taking bosentan due to the risk of hepatoxicity. Bosentan has binding affinities that are nonselective for both ET-A and ET-B. In 2001, bosentan became the first oral treatment approved by the FDA for PAH and is prescribed to be taken twice daily. ${ }^{5}$

The approval of ambrisentan did not come until 2007. Ambrisentan is taken once daily and has greater sensitivity for ET-A than it does for ET-B. Because of this, it is possible that it can modify the vasoconstriction of the smooth muscle in the vasculature without the inhibitor effects on the vasodilators. ${ }^{12}$

In 2013, macitentan was approved by the FDA. Macitentan has a greater binding affinity for ETB but can also bind to ET-A and is taken once daily. It is a derivative of bosentan with a slightly modified structure. The reason for the modification was to improve the safety and efficacy. It may be prescribed to be taken alone or in addition to phosphodiesterase 5 inhibitors and prostacyclin derivatives. ${ }^{17,18}$

\section{Phosphodiesterase 5 Inhibitors (PDE-5)}

The nitric oxide (NO) pathway is down-regulated. The cyclic GMP-mediated vasodilatory function of NO is impaired by phosphodiesterase 5 (PDE-5). PDE-5 inhibitors hinder this impairment and allow for NO-mediated vasodilation. This mechanism is dependent on NO availability. ${ }^{3,5,19}$ There are currently two PDE-5 inhibitors approved for the treatment of IPAH: sildenafil and tadalafil.

The first PDE-5 inhibitor was sildenafil, which was approved by the FDA in 2009. Sildenafil works as a vasodilator, allowing for better blood flow in the pulmonary arteries. It is also used to treat erectile dysfunction but has been shown to improve functional class, or the extent of heart failure, and improve exercise tolerance. ${ }^{5}$ Sildenafil is generally prescribed to be taken three times daily, unless dealing with a more severe case of PAH. Side effects of sildenafil include but are not limited to memory problems, back pain, loss of hearing, ringing in the ears, and/or dizziness. Physicians should be aware that when sildenafil is taken along with the ERA bosentan, the two drugs co-interact and sildenafil levels decrease while bosentan levels increase (putting patient at further risk for hepatoxicity). ${ }^{14}$ 
It wasn't until eight years later that the second PDE-5 inhibitor, tadalafil, was approved by the FDA. Tadalafil also improves exercise tolerance and slows disease progression. ${ }^{5}$ Side effects for tadalafil include upper respiratory tract infection, back pain, myalgia, cough, and/or nasal congestion. It was approved by the FDA for use starting at $40 \mathrm{mg} .{ }^{12,20}$

\section{Guanylate Cyclase Stimulators}

Extensive literature research demonstrated that the only reported FDA approved guanylate cyclase stimulator for IPAH is riociguat, approved in 2013. Riociguat side effects include gastroesophageal reflux disease, and the combination of side effects listed for the prostacyclin derivatives. The mechanisms of riociguat work by relaxing the arteries, causing the blood pressure to decrease and blood flow to increase. Riociguat is taken three times daily and was shown to have a positive effect on improving exercise tolerance. Physicians must be aware that female patients must be enrolled in Adempas Risk Evaluation and Mitigation Strategy (REMS) program to be able to receive the drug. This program sets contraception guidelines as well as regular pregnancy testing for all women taking riociguat. The reason for this is that riociguat can harm a developing fetus; therefore, women in child-bearing years must take certain precautions that are outlined in the program. ${ }^{21,22}$

Table 2 summarizes the updated medications and the pathways that they are involved in; it was created based on information in Table 1 from Ruiz, G. et. al. ${ }^{23}$

Table 2. Medication and the mechanism of action for PAH.

\begin{tabular}{r|l|l}
\multicolumn{2}{c}{ Medication } & Mechanism of Action \\
\hline Endothelin Pathway & \\
Bosentan & ERA \\
Macitentan & ERA & ERA \\
Mathway & \\
Sildenafil & PDE-5 \\
Tadalafil & PDE-5 \\
Riociguat & SGC stimulator \\
Prostacyclin Pathway & \\
Epoprostenol & Prostanoid \\
Treprostinil & Prostanoid \\
Iloprost & Prostanoid \\
Selezipag & Prostanoid
\end{tabular}

\section{Transplantations}

If medications do not work or lose efficacy, the remaining treatment option is to perform a lung or heart and lung transplant. The possible transplant types include: single lung transplant, double lung transplant, or heart and lung transplant. The median survival rate after lung transplant is 4.9 years and the median survival rate after both heart and lung transplant is 5 years. Most deaths after transplantation occur within the first year. ${ }^{24}$ Consideration of transplant is recommended early in disease progression in order to be evaluated for the transplant list and allow adequate 
survival time until a transplant becomes available. ${ }^{12}$ Limitations of transplantation include the possibility of rejection, limited number of organs that become available, and also finding the perfect match.

\section{Combination Treatments and Therapies}

Research considering the use of a combination of treatment and therapies has been limited and mostly observational. One clinical trial in 2008 showed promising results for IPAH using the combination treatment of prostacyclin derivatives and PDE inhibitors, specifically epoprostenol and sildenafil. This was a 2-arm treatment group; one arm was taking only the maximum amount of epoprostenol while the treatment group (combination group) was taking both epoprostenol and sildenafil. The results included a statistically significant 6-minute walk distance increase and a greater decrease in pulmonary arterial pressure in the combination group. ${ }^{25}$

A more recent clinical trial conducted a 3-arm clinical trial comparing ambrisentant vs. tadalfil vs. the combination of ambrisentan and tadalfil. The results of this study were published in 2015 and showed an improvement in 6-minute walk distance but there were higher numbers of adverse events. Some of the adverse events included anemia, nasal congestion, headache and peripheral edema. ${ }^{26}$ Although there are no clinical trials to highly support the use of combination medicines and therapies, physicians often prescribe blood thinners, diuretics, digoxin, and oxygen therapy in addition to the single agent treatments listed above. ${ }^{6}$ At the physician's discretion, a combination of the single agents may be prescribed. This may happen if a patient fails to respond to the first therapy and completely changing to a single agent could result in regression of current clinical status. ${ }^{12}$

\section{Discussion}

There are limitations in diagnosing and managing the disease. Because of the disease incidence being low and the signs and symptoms being nonspecific, diagnosis can be a challenge. When a patient who presents with symptoms that are nonspecific, such as dyspnea or dizziness, and other diseases have been ruled out, PAH should be considered as a diagnosis by the physician. Echocardiography, as mentioned before, is a tool used in the diagnosis process. Overreliance on this test sometimes results in misclassification and management measures of the disease. To help in selecting the right therapy and treatment of the disease, a patient should undergo right heart catheterization to get an accurate measure of the pressures and assess right ventricular function.

Education of both public and healthcare providers could help increase awareness of the disease. This education could help result in consideration of this disease more often when cardiorespiratory disorders cannot be attributed to the patient symptoms. With consideration of this disease more often, early referral is recommended of patients with suggestive symptoms or abnormal echocardiography, especially high risk population, to specialists in this field to confirm the diagnosis through right heart catheterization. Early referral could result in the patient getting the care they need quicker, leading to management of the disease which could result in better quality of life. The efficacy of these medications overall seem to improve quality of life, however, no cure has yet to be found. 


\section{Conclusion}

Over the past couple decades, the availability and quality of treatments and therapies have improved for IPAH. Although treatments and therapies have improved disease progression and survival rate, there is still no definite cure. Future research should target new classes of drugs in the effort to find a cure, as well as to explore the effectiveness of combination of treatments and therapies. Currently, there is no clear assertion as to which treatment is better; physicians should evaluate on a case-by- case basis. This review aimed to include all current FDA approved treatments and therapies for idiopathic pulmonary arterial hypertension, achieved through extensive literature research and confirmations from fda.gov. 


\section{References}

1. Baliga RS, MacAllister RJ, Hobbs AJ. New perspectives for the treatment of pulmonary hypertension. British journal of pharmacology. 2011;163(1):125-40.

2. Holland K. What is Idiopathic Pulmonary Arterial Hypertension. 2014.

3. Waxman AB, Zamanian RT. Pulmonary arterial hypertension: new insights into the optimal role of current and emerging prostacyclin therapies. The American journal of cardiology. 2013;111(5 Suppl):1A-16A; quiz 7A-9A.

4. Lai YC, Potoka KC, Champion HC, Mora AL, Gladwin MT. Pulmonary arterial hypertension: the clinical syndrome. Circulation research. 2014;115(1):115-30.

5. Bazan IS, Fares WH. Pulmonary hypertension: diagnostic and therapeutic challenges. Therapeutics and clinical risk management. 2015;11:1221-33.

6. Idiopathic Pulmonary Arterial Hypertension. Drugscom. 2016.

7. Raed A. Dweik GAH, Omar A. Minai, Adriano R. Tonelli. Pulmonary Hypertension 2011. Available from: http://www.clevelandclinicmeded.com/medicalpubs/diseasemanagement/pulmonary/pulmonaryhypertension/.

8. $\quad$ Todd Hirschtritt MKS, Nicholas S Hill Pulmonary Hypertension2008.

9. Humbert M, Sitbon O, Simonneau G. Treatment of pulmonary arterial hypertension. The New England journal of medicine. 2004;351(14):1425-36.

10. Zhang Y, He LS, Zhang G, Xu Y, Lee OO, Matsumura K, et al. The regulatory role of the NO/cGMP signal transduction cascade during larval attachment and metamorphosis of the barnacle Balanus (=Amphibalanus) amphitrite. The Journal of experimental biology. 2012;215(Pt 21):3813-22.

11. Burger CD, D'Albini L, Raspa S, Pruett JA. The evolution of prostacyclins in pulmonary arterial hypertension: from classical treatment to modern management. The American journal of managed care. 2016;22(1 Suppl):S3-15.

12. Ventetuolo CE, Klinger JR. WHO Group 1 pulmonary arterial hypertension: current and investigative therapies. Progress in cardiovascular diseases. 2012;55(2):89-103.

13. Mitchell JA, Ahmetaj-Shala B, Kirkby NS, Wright WR, Mackenzie LS, Reed DM, et al. Role of prostacyclin in pulmonary hypertension. Global cardiology science \& practice. 2014;2014(4):382-93.

14. Medicine SSo. FDA-approved Treatments for Pulmonary Hypertension. Stanford Medicine. 2016.

15. Walsh S. FDA approves new orphan drug to treat pulmonary arterial hypertension. FDA. 2015.

16. Kowalczyk A, Kleniewska P, Kolodziejczyk M, Skibska B, Goraca A. The role of endothelin-1 and endothelin receptor antagonists in inflammatory response and sepsis. Archivum immunologiae et therapiae experimentalis. 2015;63(1):41-52.

17. Kuntz M, Leiva-Juarez MM, Luthra S. Systematic Review of Randomized Controlled Trials of Endothelin Receptor Antagonists for Pulmonary Arterial Hypertension. Lung. 2016;194(5):723-32.

18. Dhillon S. Macitentan: a review of its use in patients with pulmonary arterial hypertension. Drugs. 2014;74(13):1495-507.

19. Matsubara H, Ogawa A. Treatment of idiopathic/hereditary pulmonary arterial hypertension. Journal of cardiology. 2014;64(4):243-9.

20. Galie N, Brundage BH, Ghofrani HA, Oudiz RJ, Simonneau G, Safdar Z, et al. Tadalafil therapy for pulmonary arterial hypertension. Circulation. 2009;119(22):2894-903.

21. FDA approves Adempas to treat pulmonary hypertension. FDA. 2013.

22. Ghofrani HA, Galie N, Grimminger F, Grunig E, Humbert M, Jing ZC, et al. Riociguat for the treatment of pulmonary arterial hypertension. The New England journal of medicine. 2013;369(4):330-40.

23. George Ruiz M, MBA; Gary M. Besinque, PharmD; Cassandra A. Lickert, MD; Susan Raspa, RN, BSN Combination Therapy in Pulmonary Arterial Hypertension: Is This the New Standard of Care? . 2015.

24. Schaffer JM, Singh SK, Joyce DL, Reitz BA, Robbins RC, Zamanian RT, et al. Transplantation for idiopathic pulmonary arterial hypertension: improvement in the lung allocation score era. Circulation. 2013;127(25):2503-13.

25. Simonneau G, Rubin LJ, Galie N, Barst RJ, Fleming TR, Frost AE, et al. Addition of sildenafil to longterm intravenous epoprostenol therapy in patients with pulmonary arterial hypertension: a randomized trial. Ann Intern Med. 2008;149(8):521-30.

26. Galie N, Barbera JA, Frost AE, Ghofrani HA, Hoeper MM, McLaughlin VV, et al. Initial Use of Ambrisentan plus Tadalafil in Pulmonary Arterial Hypertension. The New England journal of medicine. 2015;373(9):834-44. 